\title{
Complex Envelope Variable Approximation in Nonlinear Dynamics
}

\author{
V. V.Smirnov, L. I. Manevitch
}

We present the complex envelope variable approximation (CEVA) as a useful and compact method for analysis of essentially nonlinear dynamical systems. The basic idea is that the introduction of complex variables, which are analogues of the creation and annihilation operators in quantum mechanics, considerably simplifies the analysis of a number of nonlinear dynamical systems. The first stage of the procedure, in fact, does not require any additional assumptions, except for the proposition of the existence of a single-frequency stationary solution. This allows us to study both the stationary and nonstationary dynamics even in the cases when there are no small parameters in the initial problem. In particular, the CEVA method provides an analysis of nonlinear normal modes and their resonant interactions in discrete systems for a wide range of oscillation amplitudes. The dispersion relations depending on the oscillation amplitudes can be obtained in analytical form for both the conservative and the dissipative nonlinear lattices in the framework of the main-order approximation. In order to analyze the nonstationary dynamical processes, we suggest a new notion - the "slow" Hamiltonian, which allows us to generate the nonstationary equations in the slow time scale. The limiting phase trajectory, the bifurcations of which determine such processes as the energy localization in the nonlinear chains or the escape from the potential well under the action of external forces, can be also analyzed in the CEVA. A number of complex problems were studied earlier in the framework of various modifications of the method, but the accurate formulation of the CEVA with the step-by-step illustration is described here for the first time. In this paper we formulate the CEVA's formalism and give some nontrivial examples of its application.

Keywords: nonlinear dynamical systems, asymptotic methods, nonlinear normal modes, limiting phase trajectory, complex envelope approximation

Received April 25, 2020

Accepted August 10, 2020

The work was supported by the Russia Science Foundation (grant RSF 16-13-10302).

\author{
Valeri V.Smirnov \\ vvs@polymer.chph.ras.ru \\ Leonid I. Manevitch \\ manevitchleonid3@gmail.com \\ Federal Research Center for Chemical Physics, Russia Academy of Science \\ ul. Kosygina 4, Moscow, 119991 Russia
}

RUSSIAN JOURNAL OF NONLINEAR DYNAMICS, 2020, 16(3), 491-515 


\section{Introduction}

The modern level of scientific research and technologies more frequently leads to problems the core of which is associated with nonlinear dynamical processes. It concerns the macro- as well as micro- and nano-scale phenomena. If the first is the traditional scope of nonlinear dynamics, the second is a new impetuous developing direction of nonlinear studies. Generally speaking, nonlinear dynamics takes part in so large a number of sciences and technologies that the fact leads to formation of some interdisciplinary "nonlinear science" [1-3]. However, in spite of the fact that nonlinear dynamics is required everywhere, these problems remain very complex and are far from completion. The diversity and complexity of nonlinear dynamical problems lead to tedious and frequently ambiguous approximations. Another difficulty is due to the fact that numerical procedures allow no extrapolation of the results obtained in one problem into another. These factors combined explain why the interest in the seemingly well-studied problems still persists.

There are many analytical and numerical methods involved in the analysis of nonlinear problems [4-12]. Methods based on the asymptotic series expansion of a solution in powers of a small parameter constitute a significant part of the most widely used approaches to the study of nonlinear problems. In particular, the method of multiscale expansion is based on the separation of the time scales the ratio of which is determined by a small parameter $[12,13]$. An elementary example can be found in the beating phenomenon in the system of the weakly coupled identical oscillators. The process of energy transfer from one oscillator to another is determined by the frequency gap between in- and out-of-phase modes. In such a formulation this problem nearly relates to the slowly varying envelope approximation, which has been suggested by Van der Pol $[14,15]$. If the oscillators have nonlinear characteristics, not only the slow transfer of energy, but its capture on one of the oscillators (energy localization) becomes possible [16]. Here one should emphasize that the description of the energy transfer and localization has been made in terms of the complex representation of variables $[17,18]$ in a form that is an analogue of the secondary quantization formalism $[18,19]$. Such an approach, combined with the multiscale expansion, turns out to be very successful in the investigation of a wide class of nonlinear problems: coupled nonlinear oscillators [16, 20], forced nonlinear oscillators [21-23], energy transfer and localization in 1D nonlinear lattices $[22,24,26]$, mode coupling and energy localization in carbon nanotubes [27, 28], the synchronization of self-excited oscillators [29] and the classical analogue of the superradiant quantum transition [30], nonlinear passive control and energy sink [31,32], and the problem of rotation stability of coupled pendulums [33]. In a number of cases the complex representation of variables allows us to find a stationary singlefrequency solution (nonlinear normal modes) for the essentially nonlinear systems in analytical form without any assumptions about oscillation amplitudes $[25,26]$. Thus, a large advantage of this approach is that we obtain the main approximation which satisfies the initial essentially nonlinear problem, instead of developing an asymptotic series which is based on the linear truncation. Nonstationary dynamics can be studied in terms of slowly varying envelopes. It should be noted that no restriction on the varying amplitude of the nonstationary oscillations arises, but the main requirement is a frequency closeness of the nonstationary and stationary solutions. In particular, such conditions allow one to study the interactions of the nonlinear modes in discrete extended systems if the lengths of the latter are large enough [24-26]. In such a case, the slow time scale naturally appears from the smallness of the intermode frequency gap.

Generally speaking, the approach discussed below is a hybrid one. Actually, it is formally similar to the Van der Pol slow varying envelope approximation $[14,15]$, and is in some sense close to the harmonic balance method [8]. On the other hand, the multiple scale expansion is 
the essential constituent of the consideration of the nonstationary dynamics, but revealing the small parameter post factum makes it close to semi-inverse methods. Moreover, the procedure of constructing nonstationary equations is conceptually close to Whitham's average Lagrangian method $[43,44]$. Taking the above into account, we will refer to the approach under discussion as the "Complex Envelope Variable Approximation" (CEVA). The present work is aimed at describing the CEVA's formalism in a general case (Sections 2 and 3) and at demonstrating some examples in which the CEVA's advantages can be revealed (Section 4). Section 5 contains a short discussion and conclusions.

\section{The Complex Envelope Variable Approximation}

Let us consider the nonlinear dynamical system whose evolution is governed by the equation

$$
\frac{d^{2} u}{d t^{2}}+F(u)=0
$$

where $u$ is a function of time $t$ and $F$ is a nonlinear function of $u$. In order to analyze Eq. (2.1), we introduce the new dynamical variables

$$
\Psi=\frac{1}{\sqrt{2}}\left(\sqrt{\omega} u+i \frac{1}{\sqrt{\omega}} \frac{d u}{d t}\right)
$$

where $\omega$ is a frequency which should be determined as a function of the oscillation amplitude. The inverse transformation is well known:

$$
u=\frac{1}{\sqrt{2 \omega}}\left(\Psi+\Psi^{*}\right), \quad \frac{d u}{d t}=-i \sqrt{\frac{\omega}{2}}\left(\Psi-\Psi^{*}\right)
$$

(the asterisk denotes the complex conjugated function). Using these variables, one can rewrite Eq. (2.1) as follows:

$$
i \frac{d \Psi}{d t}-\frac{\omega}{2}\left(\Psi-\Psi^{*}\right)-\frac{1}{\sqrt{2 \omega}} F\left(\frac{1}{\sqrt{2 \omega}}\left(\Psi+\Psi^{*}\right)\right)=0 .
$$

Here our main interest is in the single-frequency stationary solution of Eq. (2.1). Therefore, we assume the function $\Psi$ to be periodic. It can be represented by a Fourier series whose first term is

$$
\Psi=\psi e^{-i \omega t},
$$

where the value of $\psi$ is supposed to be constant. Expanding the function $F$ in a Taylor series and averaging Eq. (2.4) over the period $2 \pi / \omega$, we can extract the secular term. Finally, we get the equation for the function $\psi$ in the form

$$
\frac{\omega}{2} \psi-\frac{1}{\sqrt{2 \omega}} \widetilde{\Phi}\left(\psi, \psi^{*} ; \omega\right)=0
$$

At first glance, Eq. (2.6) is not simpler than the initial one. However, for quite a number of actual systems the function $\widetilde{\Phi}$ has the noteworthy structure $[41,42]$

$$
\widetilde{\Phi}\left(\psi, \psi^{*} ; \omega\right)=\sum_{k=0}^{\infty} c_{k}\left(\sqrt{\frac{2}{\omega}}\right)^{2 k+1}|\psi|^{2 k} \psi .
$$

As is shown in the Appendix, some "good" nonlinearities admit a representation of the infinite sum in Eq. (2.7) in terms of special functions. Equation (2.6) should be considered as 
the amplitude-frequency relation if we take into account the relationship between the complex value of $\psi$ and the oscillation amplitude. The latter follows from the first of Eqs. (2.3) and expression (2.5). If $A$ is the oscillation amplitude, the absolute value of $\psi$ can be written as

$$
|\psi|=\sqrt{\frac{\omega}{2}} A .
$$

In this case, Eq. (2.6) should be written as

$$
\frac{\omega^{2}}{2} A-\Phi(A)=0
$$

This equation allows us to find the oscillation frequency $\omega$ :

$$
\omega=\sqrt{\frac{2}{A} \Phi(A)}
$$

This relation exhausts the stationary problem of the free oscillations of the system with one degree of freedom.

In order to illustrate the efficiency of this procedure, we find the amplitude-frequency relation for the most known nonlinear system - the pendulum. In this case, the function $F$ in Eq. (2.1) is $\sin u$. It was shown earlier in [22] that the respective "secular" term in Eq. (2.6) reads

$$
\frac{\omega}{2} \psi-\frac{1}{\sqrt{2 \omega}} J_{1}\left(\sqrt{\frac{2}{\omega}}|\psi|\right) \frac{\psi}{|\psi|}=0,
$$

where $J_{1}$ is a Bessel function of the first order. According to relation (2.8), the argument of the Bessel function is equal to the oscillation amplitude $A$. Therefore, expression (2.10) transforms into the form

$$
\omega=\sqrt{\frac{2}{A} J_{1}(A)} .
$$

Figure 1 shows a comparison of frequency (2.12) with the exact value $\omega=\pi / 2 K\left(\sin ^{2}(A / 2)\right)$ ( $K$ is the full elliptic integral of the first kind).

Analyzing Fig. 1, one can conclude that approximation (2.12) is good enough in a wide interval of the oscillation amplitudes except in a vicinity of the limiting value $A=\pi$. It is the expected result because the motion with the amplitude $A=\pi$ corresponds to the separatrix and evidently does not belong to the category of the single-frequency solutions (2.5).

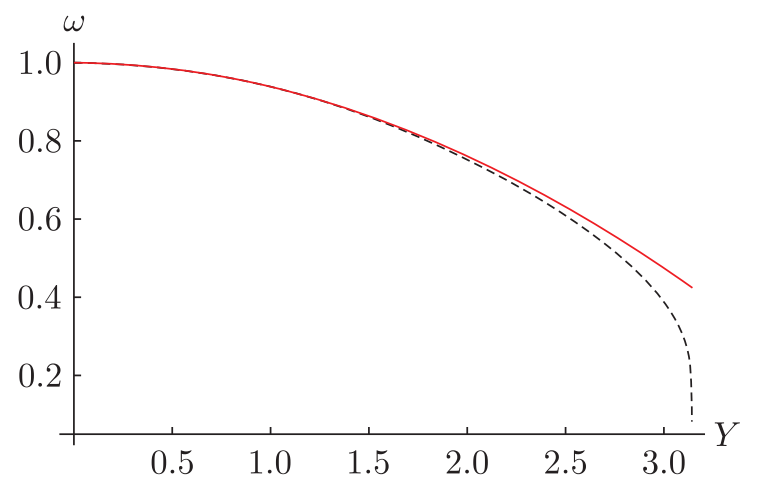

Fig. 1. The amplitude-frequency dependence in accordance with Eq. (2.12) (red solid curve) and the exact value $\omega_{0}$ (black dashed curve). 
It should be noted that the procedures discussed above are intended for finding the mainorder approximation. Naturally, no small parameter is required in order to do it.

However, if the initial problem contains no small parameter, a natural question arises what the error of the main approximation frequency is and in what manner we can correct it? At first glance, the procedure discussed above allows no estimate of the accuracy and the range of applicability of the results. In fact, if there is no small parameter, it is impossible to estimate the value of the omitted terms. In this case we can use another approach: let us assume that the next term in the Fourier expansion is small enough. Under this condition we can calculate its amplitude in the smallest order. Then we should estimate the relative value of the correction and thus check the validity of our assumption. In what follows we consider two examples of such estimations: for systems with symmetric and asymmetric potential functions.

So, we start from the oscillations of the pendulum as an example of the symmetric potential. We need to add the next harmonic to the representation (2.5) and estimate the effects of this modification. In order to keep the oscillation amplitude constant, we write the complex function $\Psi$ as follows:

$$
\Psi=\psi\left((1-\eta) e^{-i \omega t}+\eta e^{-3 i \omega t}\right) .
$$

The function $F$ in equation (2.1) is $\sin u$ and has to be expanded in a Taylor series:

$$
\sin u=\sum_{k=0}^{\infty} \frac{(-1)^{k}}{(2 k+1) !} u^{(2 k+1)} .
$$

Assuming the amplitude of the third harmonics to be small $(\eta \ll 1)$, we should keep only the corresponding linear terms in expansion (2.14). In this case, we can write

$$
\begin{gathered}
i \frac{d \Psi}{d t}-\frac{\omega}{2}\left(\Psi-\Psi^{*}\right)-\frac{1}{\sqrt{2 \omega}} \sum_{k=0}^{\infty} \frac{(-1)^{k}}{(2 k+1) !}\left(\frac{1}{\sqrt{2 \omega}}\right)^{2 k+1}\left(\Psi+\Psi^{*}\right)^{2 k+1} \approx \\
i \frac{d}{d t} \psi\left((1-\eta) e^{-i \omega t}+\eta e^{-3 i \omega t}\right)-\frac{\omega}{2} \psi\left((1-\eta) e^{-i \omega t}+\eta e^{-3 i \omega t}\right)- \\
\frac{\omega}{2} \psi^{*}\left((1-\eta) e^{i \omega t}+\eta e^{3 i \omega t}\right)-\frac{1}{\sqrt{2 \omega}} \sum_{k=0}^{\infty} \frac{(-1)^{k}}{(\sqrt{2 \omega})^{2 k+1}} \frac{(1-\eta)^{2 k}\left(\psi e^{-i \omega t}+\psi^{*} e^{i \omega t}\right)^{2 k}}{(2 k) !} \times \\
\left(\frac{(1-\eta)\left(\psi e^{-i \omega t}+\psi^{*} e^{i \omega t}\right)}{2 k+1}+\eta\left(\psi e^{-3 i \omega t}+\psi^{*} e^{3 i \omega t}\right)\right)=0 .
\end{gathered}
$$

Multiplying Eq. (2.15) by $\exp (-i \omega t)$ or $\exp (-3 i \omega t)$ and averaging over the period $2 \pi / \omega$, we can perform the summation and get the equations which determine the "weight" of the third harmonic $\eta$ and the new frequency $\omega$. These equations are very cumbersome, but taking into account relation (2.8), which determines the relation between the complex value of $\psi$ and the real amplitude of the oscillations, we can formulate them as follows:

$$
\begin{gathered}
\omega^{2}-\frac{2}{A} J_{1}(A)-\eta\left(\omega^{2}-\frac{8}{A} J_{1}(A)+\frac{24}{A^{2}} J_{2}(A)\right)=0 \\
\left(A^{2}-8\right) J_{1}(A)+4 A J_{0}(A)- \\
\frac{\eta}{2 A^{2}}\left(5 A^{5} \omega^{2}+120\left(A^{2}-16\right) A J_{0}(A)+8\left(A^{4}-90 A^{2}+480\right) J_{1}(A)\right)=0 .
\end{gathered}
$$

These equations can be solved analytically. Figure 2 shows frequency $\omega$ and relative amplitude $\eta$ as the functions of oscillation amplitude $A$. 


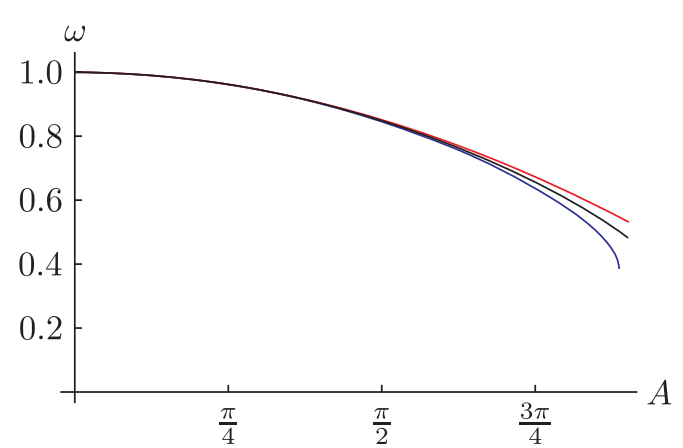

(a)

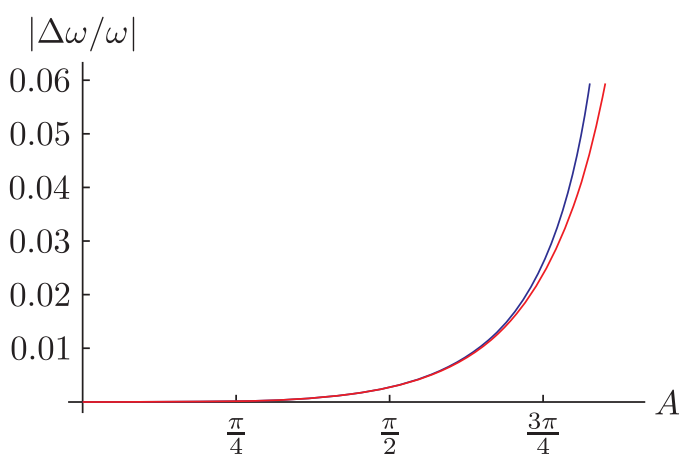

(b)

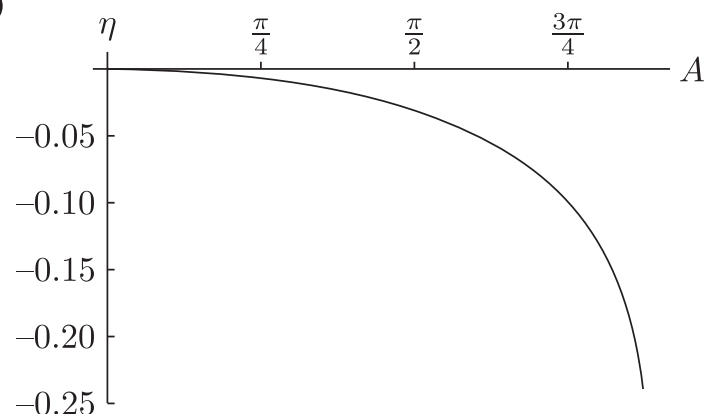

(c)

Fig. 2. (a) The black curve shows the exact frequency of pendulum oscillations vs their amplitude $A$, while the red and blue curves correspond to the main approximation frequency (2.10) and the first correction to it (2.16), respectively. (b) The relative error for the curves, which are shown in panel (a) (the colors are the same). (c) The relative "weight" of the third harmonic $\eta$ vs oscillation amplitude $A$.

The analysis of Fig. 2 shows that the effect of the third harmonic on the oscillation frequency enlarges the relative error, but it corrects the frequency of the main approximation in the "right" direction. Moreover, in accordance with our assumptions, the relative value of the third harmonic is small everywhere except in the narrow band near the limit oscillation amplitude (see panel (c) of Fig. 2). Generally speaking, the procedures (2.13)-(2.15) show in what manner we can continue the expansion of the complex function $\Psi$, taking into account the higher order of the harmonic amplitudes $\left(\eta^{n}\right)$ or the next harmonics themselves $\left(\sim e^{-i k \omega t}\right)$.

The next problem, which cannot be solved in the main order of the CEVA, concerns the oscillations in the asymmetric potential well. Actually, the averaging equations for the complex function $\Psi$, if it is represented in the form (2.5), preserve only odd terms in the Taylor series of the function $F$ (see Eq. (2.7)). So, if the potential function contains the odd terms of the dependent variable, their contribution to the function $\widetilde{\Phi}$ will be lost. Let us consider the simple asymmetric potential

The equation of motion is

$$
U(u)=\frac{\Omega^{2}}{2} u^{2}+\frac{\beta}{3} u^{3} .
$$

$$
\frac{d^{2} u}{d t^{2}}+\Omega^{2} u+\beta u^{2}=0 .
$$

After transition to the complex variables we choose the complex function $\Psi$ in the form (2.5). Substituting it into Eq. (2.18) and averaging over the period, we obtain

$$
\frac{\omega}{2} \psi-\frac{\Omega^{2}}{2 \omega} \psi=0
$$


This equation has the obvious solution $\omega=\Omega$, which does not depend on the oscillation amplitude and does not represent the features of the potential function (2.17). In particular, the oscillations in the asymmetric potential are characterized by the nonzero average displacement, while the mean of the function $\Psi(2.5)$ is zero. Moreover, the frequency of oscillations in the potential well (2.18) essentially depends on their amplitude and it decreases up to zero when the energy reaches the value $\Omega^{6} / 2 \beta^{2}$. Taking the above into account, we represent the function $\Psi$ as follows:

$$
\Psi=\psi\left(\varepsilon+(1-\varepsilon-\eta) e^{-i \omega t}+\eta e^{-2 i \omega t}\right) .
$$

We will suppose that the "weights" of zero and second harmonics ( $\varepsilon$ and $\eta$, respectively) are small:

$$
\varepsilon \ll 1, \quad \eta \ll 1 .
$$

In order to make the procedure transparent, we write the full equation for the function $\psi$ once again

$$
\begin{aligned}
& \frac{\omega}{2} \psi\left(\varepsilon+(1-\eta-\varepsilon) e^{-i t \omega}+\eta e^{-2 i t \omega}\right)-\frac{\omega}{2} \psi^{*}\left(\varepsilon+(1-\eta-\varepsilon) e^{i t \omega}+\eta e^{2 i t \omega}\right)- \\
& \frac{\Omega^{2}}{2 \omega}\left(\psi\left(\varepsilon+(1-\eta-\varepsilon) e^{-i t \omega}+\eta e^{-2 i t \omega}\right)+\psi^{*}\left(\varepsilon+(1-\eta-\varepsilon) e^{i t \omega}+\eta e^{2 i t \omega}\right)\right)- \\
& \frac{\beta}{(2 \omega)^{3 / 2}}\left(\psi\left(\varepsilon+(1-\eta-\varepsilon) e^{-i t \omega}+\eta e^{-2 i t \omega}\right)+\psi^{*}\left(\varepsilon+(1-\eta-\varepsilon) e^{i t \omega}+\eta e^{2 i t \omega}\right)\right)^{2}=0 .
\end{aligned}
$$

In order to obtain the equations that determine the values of $\omega, \varepsilon$ and $\eta$, one should multiply Eq. (2.22) by unity, $e^{i \omega t}$ and $e^{2 i \omega t}$ and perform averaging over the period $2 \pi / \omega$. Keeping in mind relation (2.8) between the oscillation amplitude and the modulus of the complex function $\psi$, we get three equations:

$$
\begin{aligned}
A \beta(1-2 \eta-2 \epsilon)+2 \Omega^{2} \epsilon & =0, \\
A \beta(\eta+2 \epsilon)-\omega^{2}+\Omega^{2} & =0, \\
A \beta(1-2 \eta-2 \epsilon)+2 \eta\left(\Omega^{2}-3 \omega^{2}\right) & =0 .
\end{aligned}
$$

These equations have multiple roots, but it is the root with the asymptotics $\omega \rightarrow 1, \varepsilon \rightarrow 0$ and $\eta \rightarrow 0$ as $A \rightarrow 0$ that is physically meaningful. Figure 3 shows the oscillation frequencies and the relative values of zero and second harmonics as the functions of amplitude $A$ for the potential (2.17). The black curve shows the exact value of oscillation frequency $\omega$ and the red curve is calculated as the solution of Eqs. (2.24). One can see that counting the zero harmonic is only reasonable for small amplitudes $(A \leqslant 0.3)$, but the increasing amplitude leads to the necessity of the second harmonic. In spite of the fact that the approximate frequency does not exhibit the right tendency, its relative difference from the exact value does not exceed 5 per cent up to the oscillation amplitude $A \sim 0.7 A_{\max }$. Panel (b) of Fig. 3 shows the relative contribution of zero and second harmonics. Figure 3(c) presents a comparison of the results of the direct numerical integration of Eq. (2.18) and the calculations which have been performed within the framework of the CEVA. The initial conditions correspond to the pulse with velocity $V$, which is equal to one half of the maximal one. The latter is calculated from the energy of the potential's maximum and it is $V_{\max }=\Omega^{3} / \sqrt{3} \beta$. The amplitude of the oscillations was calculated as half the distance between the return points. The calculated and measured (dimensionless) frequencies are 0.963 and 0.961 , respectively.

We conclude this section by referring to the extension of the single frequency approximation (2.5), which can be found in [31].

RUSSIAN JOURNAL OF NONLINEAR DYNAMICS, 2020, 16(3), 491-515 


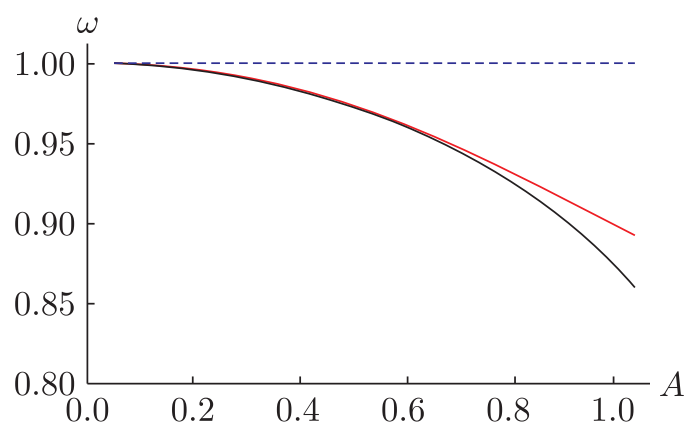

(a)

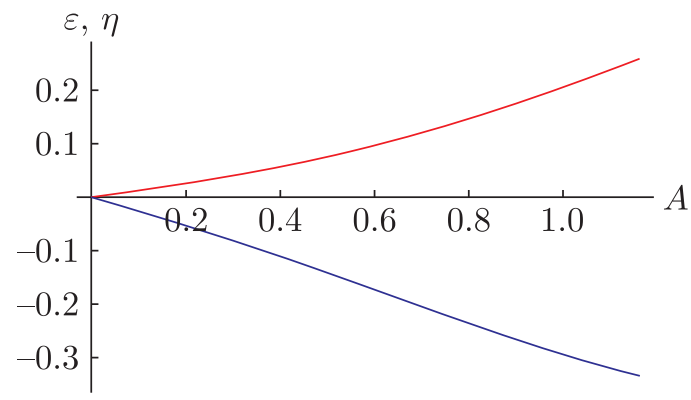

(b)

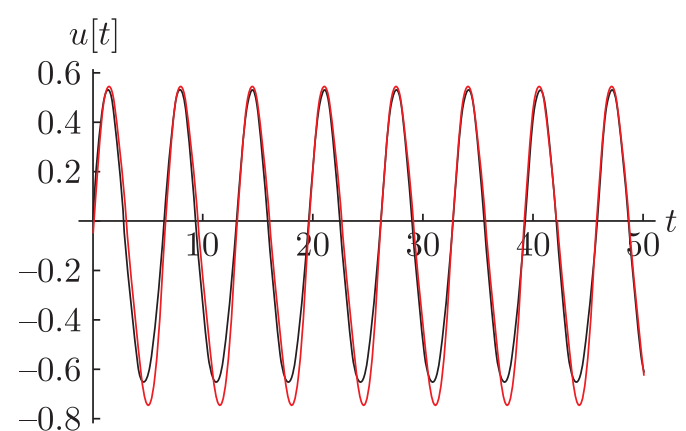

(c)

Fig. 3. a) The black curve shows the exact frequency of the oscillations in potential well (2.17) vs their amplitude $A$; blue dashed line shows the main approximation frequency; the red curve corresponds to the approximation frequency with zero and second harmonics. b) The relative values of zero $(\varepsilon)$ and second $(\eta)$ harmonics are shown by blue and red curves, respectively. c) The comparison of the numerical integration of Eq. (2.18) (black curve) and calculation of the oscillation in the framework of the CEVA. The initial conditions correspond to zero displacement and one half of the maximal velocity $V_{\max }=\Omega^{3} / \sqrt{3} \beta$. Potential's parameters: $\Omega=1, \beta=0.5$.

\section{Nonstationary Dynamics with Complex Envelope Variable Approximation}

In the previous section we considered the main-order approximation and the next order corrections to it. However, these corrections, as well as the main approximation itself, remain in the scope of the stationary dynamical problem, i.e., the function $\Psi$ with or without high harmonic terms describes stationary oscillations only. Therefore, a natural question arises in what manner we can expand the CEVA to describe the nonstationary processes? One should note that by nonstationary processes we mean changes in the amplitude and/or the frequency of oscillations, which is slow enough compared to the period of oscillations. In nonlinear problems such variations can result from the external perturbation or weak interaction of the nonlinear normal modes. The multiscale expansion method $[12,13]$ is based on the introduction of the hierarchy of times which are scaled by the order of the small parameter $\epsilon$. In the framework of this method, the processes with times $\sim \epsilon^{n}$ with $n \geqslant 2$ are considered as the nonstationary ones. The classical Bogoliubov-Krylov method [4] consists in the construction of governing equations for the amplitude and phase of the main-order approximation, the right-hand sides of which are the series on the small parameter. Because the right-hand sides do not contain any terms $\sim O(1)$, the variations of the amplitude and phase variables turn out to be slow. 
However, we want to understand what we have to do if the initial problem, as well as the main-order approximation, does not contain any small parameters? We cannot say a priori what factor will determine the varying oscillation amplitude. In order to avoid these difficulties, we will use an approach which is an analogue of the average Lagrangian method in the dispersive waves theory due to Whitham [43, 44]. We will define the average Hamiltonian, which is formulated in terms of complex envelope variables and the variation of which allows us to construct the nonstationary equations in the slow time scale.

Before starting the discussion of the application of the CEVA to nonstationary dynamics, we would like to consider an elementary example aimed at demonstrating the formal procedure and to make the physical content clear. Let us consider a simple linear oscillator whose Hamiltonian function is written in the form

$$
H_{0}=\frac{1}{2}\left(\frac{d u}{d t}\right)^{2}+\frac{\omega_{0}^{2}}{2} u^{2}
$$

This function can be represented in terms of the complex variables (2.2) as follows:

$$
H_{0}=-\frac{\omega}{4}\left(\Psi-\Psi^{*}\right)^{2}+\frac{\omega_{0}^{2}}{4 \omega}\left(\Psi+\Psi^{*}\right)^{2}
$$

Substituting the form (2.5) for the function $\Psi$ into the Hamiltonian (3.2) and averaging over the period, we get

$$
\left\langle H_{0}\right\rangle=\frac{\omega}{2}|\psi|^{2}+\frac{\omega_{0}^{2}}{2 \omega}|\psi|^{2}=\frac{\omega_{0}^{2}+\omega^{2}}{2 \omega}|\psi|^{2} .
$$

This expression shows the average energy of the oscillations of the linear oscillator. We know that the self-excited oscillations of the linear oscillator are characterized by the frequency $\omega=\omega_{0}$. Taking relation (2.8) into account, we get the standard value

$$
\left\langle H_{0}\right\rangle=\omega_{0}|\psi|^{2}=\frac{\omega_{0}^{2}}{2} A^{2} .
$$

On the other hand, the linear version of Eq. (2.6) can be associated with the Hamiltonian function

$$
\mathscr{H}=\frac{\omega_{0}^{2}}{2 \omega}|\psi|^{2}-\frac{\omega}{2}|\psi|^{2}=\frac{\omega_{0}^{2}-\omega^{2}}{2 \omega}|\psi|^{2} .
$$

There is an essential difference between the functions (3.3) and (3.5), in spite of the fact that they have to describe the same process. If we suppose $\omega=\omega_{0}$ in expression (3.5), we get $\mathscr{H}=0$. The relation between $\left\langle H_{0}\right\rangle$ and $\mathscr{H}$ can be written as

$$
\mathscr{H}=\left\langle H_{0}\right\rangle-\omega|\psi|^{2}
$$

i.e., the function $\mathscr{H}$ shows the difference between the average energy of the system $\left\langle H_{0}\right\rangle$ and the energy of the oscillations with frequency $\omega$. Naturally, for the stationary natural oscillations this value should be zero.

However, the function $\mathscr{H}$ has very important properties. Its variations give us the frequency characteristic of the system. In fact, the equality

$$
\frac{\partial \mathscr{H}}{\partial \psi^{*}}=\frac{\omega_{0}^{2}-\omega^{2}}{2 \omega} \psi=0
$$

leads immediately to the relation $\omega=\omega_{0}$. It is important to emphasize that the Hamiltonians $\mathscr{H}$ and $\left\langle H_{0}\right\rangle$ do not contain any variables which are the function of the "fast" time $t$. This means 
that we are in a phase space where the processes with the specific time $\sim 1 / \omega$ are exhibited only via their frequency $\omega$, which parameterizes the function $\mathscr{H}$. So, if any changes in the function $\psi$ are possible, they have to be very slow compared to the oscillations with frequency $\omega$.

Returning to the nonlinear problem (2.6), we can write the corresponding Hamiltonian function as follows:

$$
\mathscr{H}=-\frac{\omega}{2}|\psi|^{2}+G(\psi ; \omega),
$$

where the function $\widetilde{\Phi}$ is coupled with $G$ by the relation

$$
\widetilde{\Phi}=\frac{\partial G}{\partial \psi^{*}} .
$$

The variation of the function (3.8) with respect to $\psi^{*}$ gives us the amplitude-frequency relation (or the dispersion relations for the multiparticle system) for the steady-state oscillations of the system (the nonlinear normal modes). Symbolically speaking, extracting the energy of fast motion from the Hamiltonian function $\left\langle H_{0}\right\rangle$, we can reveal the processes in the slow time scale more graphically. In order to avoid any ambiguity in what follows, we will refer to the Hamiltonian function (3.8) as the "slow Hamiltonian".

However, we can suppose that the Hamiltonian (3.8) is appropriate for analysis of weakly disturbed steady-state oscillations, which lead to nonstationary dynamics. In order to do it, one should write the equations of motion as follows:

$$
i \frac{d \psi}{d \tau}=\frac{\partial \mathscr{H}}{\partial \psi^{*}} .
$$

This leads to the time-dependent version of Eq. (2.6):

$$
i \frac{d \psi}{d \tau}+\frac{\omega}{2} \psi-\frac{1}{\sqrt{2 \omega}} \widetilde{\Phi}(\psi ; \omega)=0 .
$$

Here we denote by $\tau$ the time variable in order to emphasize its difference from the "fast" time $t$.

Really, it is easy to show that the evolution of the function $\psi$ takes place in the slow time scale. Let us consider a small disturbance of the eigen oscillations, $\psi=\psi_{0}+\delta \psi$. In this case we can expand the right-hand side of Eq. (3.10) in a series on $\delta \psi$ :

$$
\frac{\partial \mathscr{H}}{\partial \psi^{*}} \approx \frac{\partial \mathscr{H}}{\partial \psi^{*}}{ }_{\mid \psi^{*}=\psi_{0}^{*}}+{\frac{\partial^{2} \mathscr{H}}{\partial \psi^{* 2}}}_{\mid \psi^{*}=\psi_{0}^{*}} \delta \psi^{*}+\ldots
$$

The first term on the right-hand side of Eq. (3.12) disappears for the natural oscillations $\psi_{0}^{*}$. In order to estimate the second term, one should remember that the derivative of the energy of the stationary oscillation with respect to the occupation number $X=|\psi|^{2}$ is equal to the oscillation frequency [18]:

$$
\omega=\frac{\partial E}{\partial X} .
$$

This correlates well with the quantum mechanical rule $E=\omega X$. In this case, the factor in front of $\delta \psi^{*}$ is proportional to the derivative of the frequency with respect to the occupation number, i.e., the amplitude of the oscillations:

$$
\frac{\partial^{2} \mathscr{H}}{\partial \psi^{* 2}} \sim \frac{\partial \omega}{\partial X}
$$


So, we can conclude that, if the dependence of the energy on the oscillation amplitude is smooth enough, the nonstationary evolution will be slow.

The disturbance $\delta \psi$ can be an amplitude variation or contain the phase deviation. Such processes can arise in the case of resonant interaction of the nonlinear normal modes or coupled nonlinear oscillators, although the bonds between oscillators are not necessarily weak. The resonant conditions can occur due to the nontrivial dependence of the frequency on the amplitude or the wave number. In particular, the total degeneration of the nonlinear normal modes appears in the system of the interacting dipoles with antiferromagnetic arrangement under the action of the external field [45].

Let us use the pendulum in order to make certain that Eqs. (3.11) do describe the slow-time evolution of the system under a small disturbance of the pendulum's stationary oscillations. The question is what nonstationary dynamics is possible in the single pendulum motion without any dissipative effects. In accordance with relation (2.12), any oscillation amplitude corresponds to the unique frequency and any disturbance leads to a transition to a new stationary oscillation state. Therefore, except during the transition time (i.e., the time of action of the external force), the pendulum oscillations are stationary. In order to demonstrate in what manner we can observe the nonstationary dynamics of the pendulum, we propose the next conceptual experiment. Consider two identical noncoupled pendulums, which we refer to as the "reference pendulum" (RP) and the "target pendulum" (TP). We place the observer on the first pendulum (RP). Assume that at time $\tau=0$ the pendulums' states are identical. In this case, the relative positions of the pendulum are fixed and the observer will observe a stationary configuration. (Even if the initial phase shift occurs, this does not change.) At time $\tau=\tau_{1}$ we disturb the TP's oscillations (for example, we can turn on the friction for a short time). As a result, the TP transits into a new oscillating stationary state with a new amplitude and frequency, the relation between which is determined by Eq. (2.12). However, these new oscillations of the TP are not stationary from the viewpoint of the observer, who is on the RP. The phase shift between the oscillations of RP and $\mathrm{TP}$ will change continuously with the velocity, which is equal to the frequency difference. So, in spite of the fact that both oscillation states are stationary, the second looks nonstationary from the viewpoint of the first.

It is convenient to introduce a polar representation of the function $\psi$ for the pendulum motion

$$
\psi=a e^{i \delta}
$$

and write the reduced Hamiltonian function as

$$
\mathscr{H}=-\frac{\omega}{2} a^{2}-J_{0}\left(\sqrt{\frac{2}{\omega}} a\right) .
$$

Let $\psi=(a+\alpha) e^{i \delta}$, where $a$ is a solution of Eq. (2.11), $\omega$ is determined by Eq. (2.12), and $\alpha \ll a$ is a disturbance. Taking Eqs. (3.10) into account, we get

$$
\begin{gathered}
\frac{d \alpha}{d \tau}=0 \\
\frac{d \delta}{d \tau} \approx \frac{1}{a}\left(\frac{\omega}{2} a-\frac{1}{\sqrt{2 \omega}} J_{1}\left(\sqrt{\frac{2}{\omega}} a\right)\right)+\frac{1}{\omega a} J_{2}\left(\sqrt{\frac{2}{\omega}} a\right) \alpha+\left(\frac{J_{1}\left(\sqrt{\frac{2}{\omega}} a\right)}{\sqrt{2} a \omega^{3 / 2}}-\frac{3 J_{2}\left(\sqrt{\frac{2}{\omega}} a\right)}{2 a^{2} \omega}\right) \alpha^{2} .
\end{gathered}
$$

The first of Eqs. (3.17) shows that the amplitude of the disturbed motion does not change. The first term on the right-hand side of Eqs. (3.17) is equal to zero, and the variation of the phase $\delta$ 
turns out to be proportional to $\Delta \omega \sim \alpha d \omega / d a$. Since $\alpha=$ const, it follows that the disturbed solution runs off with constant velocity from the initial solution: $\delta \sim \Delta \omega \tau$. It should be emphasized that the time scale of the variable $\tau$ is controlled by the smallness of the right-hand side of Eqs. (3.17). The latter can be determined by the smallness of either the disturbance's amplitude $(\alpha \ll a)$ or the flatness of the oscillation frequency $(d \omega / d a \ll \omega / a)$ if the amplitude disturbance is not small.

As a conclusion of this section, let us check that Eqs. (3.17) correctly predict the frequency changing in the limit of small amplitude $a$. Assuming $a \rightarrow 0$ and $\omega \rightarrow 1$, and taking into account the relations between the modulus of the complex function and the oscillations' amplitude, we get

$$
\frac{d \delta}{d \tau} \approx \frac{\alpha^{2}}{8}=\frac{A^{2}}{16}
$$

The sign of the correction is positive because the solution (2.5) contains $e^{-i \omega t}$. The correction (3.18) agrees with the expansion of exact pendulum frequency $\omega \approx 1-A^{2} / 16$.

In the next section we consider some examples and generalizations of the procedures discussed above. Before doing this, we formulate the standard steps for development of the evolution equations within the framework of the CEVA:

i) introduce the complex variables $(2.2)$;

ii) rewrite the initial equation (2.1) in terms of the complex variables;

iii) extract the secular term for the single-frequency solution (2.5);

iv) use relation (2.8) in order to determine the stationary amplitude-frequency relation;

v) find the slow Hamiltonian function corresponding to the stationary solution;

vi) develop the nonstationary equations in accordance with relations (3.10).

\section{Examples and Applications}

\subsection{Forced damped oscillations of the pendulum}

The effect of the external forcing and dissipative processes often plays an important role in the dynamics of the nonlinear systems. Therefore, we start this section by considering the forced oscillations of the pendulum with viscous friction. Suppose that the pendulum is acted upon by an external field $P(t)=f \cos \omega t$. The stationary equation of motion can be written in terms of the complex variable $\psi$ as follows:

$$
\frac{\omega}{2} \psi-\frac{1}{\sqrt{2 \omega}} J_{1}\left(\sqrt{\frac{2}{\omega}}|\psi|\right) \frac{\psi}{|\psi|}+i \frac{\nu}{2} \psi=-\frac{f}{2 \sqrt{2 \omega}},
$$

where $\nu$ is the coefficient of viscous friction. Using relation (2.8), it is easy to find the amplitudefrequency relation for the nondissipative system $(\nu=0)$ :

$$
\omega^{2}=\frac{2}{A}\left(J_{1}(A)-f\right) .
$$

However, the function $\psi$ becomes complex for nonzero friction. Assuming $\psi=x+i y$, we need to separate the real and imaginary parts of Eq. (4.1). After some manipulations we can write 
the amplitude-frequency relation in the form

$$
\begin{aligned}
\frac{2}{\omega}\left(x^{2}+y^{2}\right) & =A^{2}=\frac{f^{2}}{\nu^{2} \omega^{2}+\left(\omega^{2}-\Omega^{2}\right)^{2}}, \\
\frac{y}{x}=\tan \delta & =-\frac{\nu \omega}{\omega^{2}-\Omega^{2}},
\end{aligned}
$$

where $\Omega=\Omega(A)=\sqrt{2 J_{1}(A) / A}$ is the frequency of free oscillations with amplitude $A$. It should be noted that the first of Eqs. (4.3) is a transcendent equation with respect to amplitude $A$. It can be solved numerically and the result is represented in Fig. 4.

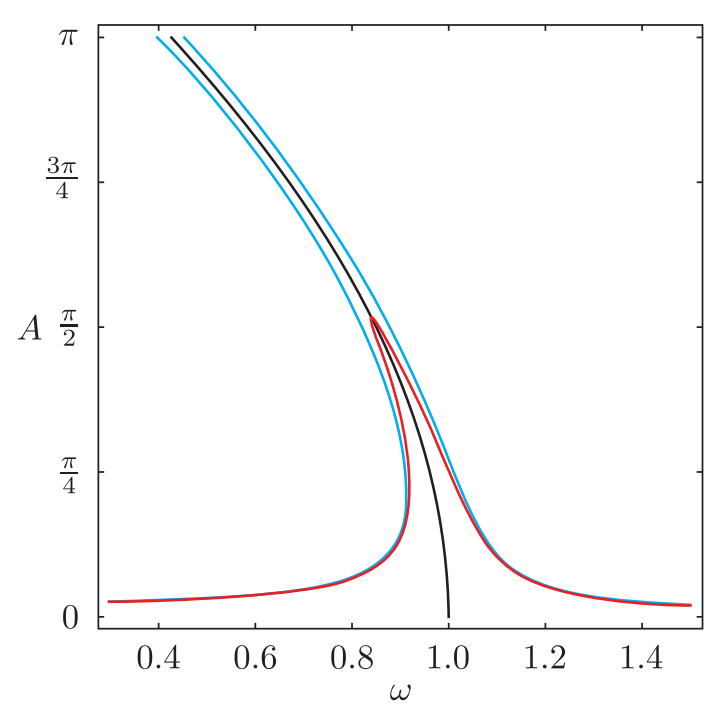

Fig. 4. Amplitude-frequency relations (2.12), (4.2), (4.3) for the free and forced oscillations of the pendulum without and with friction (black, blue and red curves, respectively). Amplitude of the external force $f=0.075$ and friction $\nu=0.055$.

It is noteworthy that the amplitude-frequency relation (4.3) looks absolutely similar to its linear analogue, with the difference that the frequency of nonlinear free oscillations with amplitude $A$ plays the role of the eigenfrequency of the linear oscillator. The expression for phase shift $\delta$ also has the same form as that for the linear system. We would like to emphasize that equations (4.3) are general in character, which is confirmed by a comparison with the amplitude-frequency relation obtained in the classical book by Bogoliubov and Mitropolsky [4]. Actually, the first of Eqs. (4.3) coincides absolutely with the expression obtained in this book if we replace the frequency $\Omega$ in (4.3) with the oscillation frequency of the nonlinear oscillator. Thus, this important result demonstrates the efficiency of application of the CEVA to analysis of the forced oscillations.

\subsection{Escape from a potential well}

We illustrate the nonstationary nonlinear dynamics by considering the escape from the potential well under the action of a single-frequency external field $P=f \cos \omega t$ without friction. The transition of the pendulum from oscillations to rotation gives a very clear example of such processes. 
Writing the function $\psi$ in polar form, we can separate the equations for the amplitude $a$ and phase $\delta$ :

$$
\begin{gathered}
\frac{d a}{d \tau}=\frac{f \sin (\delta)}{2 \sqrt{2} \sqrt{\omega}} \\
a \frac{d \delta}{d \tau}=\frac{\omega}{2} a-\frac{1}{\sqrt{2 \omega}} J_{1}(a)+\frac{f \cos (\delta)}{2 \sqrt{2 \omega}} .
\end{gathered}
$$

From Eqs. (4.4) we find immediately that the energy of forced oscillations reads

$$
\mathscr{H}_{f}=-\frac{\omega}{2} a^{2}-J_{0}\left(\sqrt{\frac{2}{\omega}} a\right)+\frac{a f \cos \delta}{\sqrt{2 \omega}} .
$$

We would like to find out what combinations of the force's frequency $\omega$ and amplitude $f$ lead to the rotation of the pendulum if the initial conditions are zero. Such a problem is nontrivial even in the case of a simple parabolic potential [38]. In order to determine the boundaries of oscillations in the plane "frequency - force amplitude", one should analyze possible nonstationary trajectories corresponding to the Hamiltonian form (4.5). Let us consider the phase space in the terms $\{a, \delta\}$, assuming the frequency $\omega$ and the force amplitude $f$ to be the parameters. Because the Hamiltonian (4.5) does not contain the variables which depend on the "fast" time $t$, the stationary oscillations correspond to the stationary points on the phase plane $\{\delta, a\}$. There are three stationary states in the low-frequency region in Fig. 4, and only one stationary state occurs if the frequency is larger than some value $\omega_{*}$. The corresponding amplitude can be found as the root of the equation

$$
\frac{d \omega}{d A}=\frac{f-A J_{2}(A)}{\sqrt{2} A^{3 / 2} \sqrt{J_{1}(A)-f}}=0 .
$$

Figure 5 shows the phase portraits of the system (4.5) with different values of frequency $\omega$ and the constant value of the force amplitude $f$.

Figure $5 \mathrm{a}$ represents the phase portrait with a single stationary state at the phase $\delta=0$. The thick blue curve, which passes through the zero-valued amplitude, separates the trajectories closed around the stationary point from the transit-time trajectories. These trajectories are called the limiting phase trajectories (LPT). In the problem under consideration the LPT describes the escape from the potential well if the maximum of the LPT exceeds the limiting angle of the oscillations (i.e., $\pi$ ). Such a case is observed in panel (b) of Fig. 5. The value of the threshold frequency can be evaluated from the condition

$$
\mathscr{H}_{f}\left(a=0, \delta=\frac{\pi}{2}\right)=\mathscr{H}_{f}\left(a=\sqrt{\frac{\omega}{2}} \pi, \delta=0\right) .
$$

This condition corresponds to the high-frequency boundary of the escape from the well. It should be noted that the phase portrait in panel (b) contains three stationary points, therefore, the corresponding frequency is smaller than the frequency $\omega_{*}$ mentioned above. By decreasing frequency $\omega$ we can obtain the phase portrait depicted in panel (c) of Fig. 5. The specific feature of this phase portrait is that the LPT coincides with the separatrix crossing the unstable stationary point at $\delta=\pi$. There is no possibility of escaping the potential well at this frequency and at smaller ones (see Fig. 5d) because the trajectory which starts at $a=0$ cannot reach 


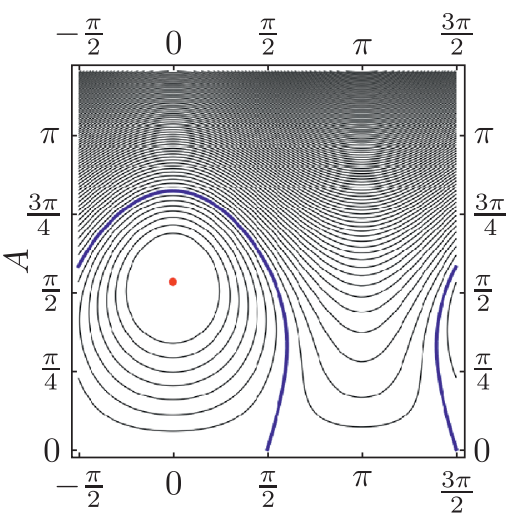

(a)

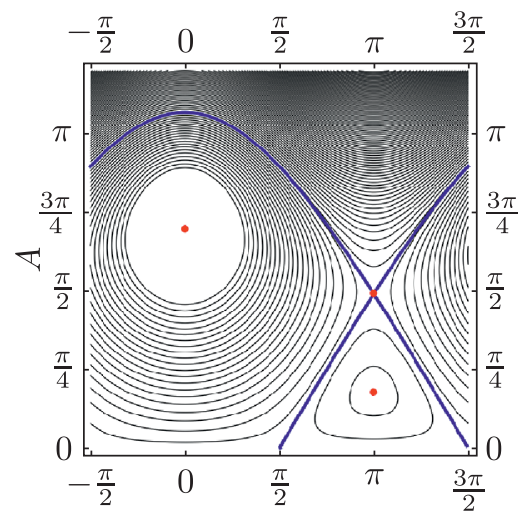

(c)

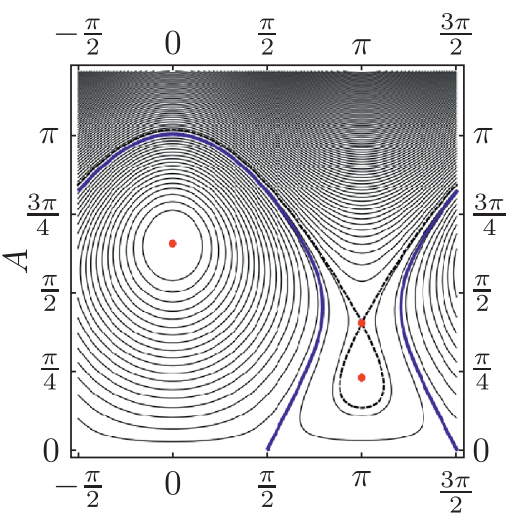

(b)

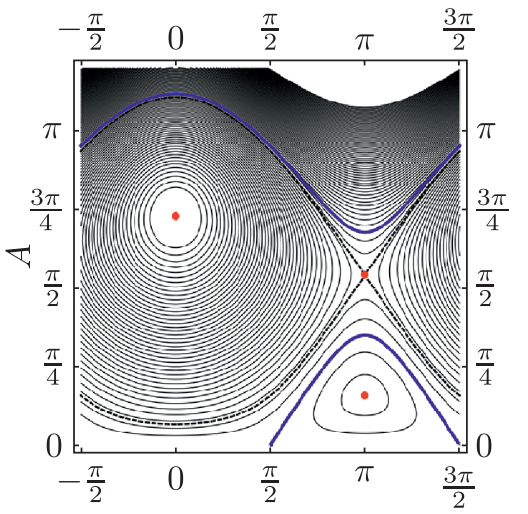

(d)

Fig. 5. The phase portraits of system (4.5) at force amplitude $f=0.2$ and different values of frequency $\omega$. Panels (a)-(d) correspond to $\omega=0.9,0.81,0.776,0.75$, respectively. The limiting phase trajectories and the separatrix are shown as thick blue and black dashed curves, respectively. Red points correspond to the stationary states.

the limiting angle $\pi$. The frequency corresponding to the phase portrait in panel (c) can be evaluated by solving the equation

$$
\mathscr{H}_{f}\left(a=0, \delta=\frac{\pi}{2}\right)=\mathscr{H}_{f}\left(a=a_{u}, \delta=\pi\right)
$$

where $a_{u}$ corresponds to the unstable stationary point.

Thus, solving Eqs. (4.7) and (4.8), we can determine the domain of the force's frequency and amplitudes, where the escape from the potential well is possible. It is noteworthy that, in order to do this, we do not need to solve the nonstationary equations (4.4), but we can find the domain's boundaries by analyzing the variation of the phase portrait at various values of $f$ and $\omega$.

Nevertheless, if we want to estimate the time of the escape from the well, we need to integrate the nonstationary equation along the LPT. Actually, the escape time can be estimated as follows:

$$
T=\int_{0}^{T} d \tau=\int_{0}^{\sqrt{\frac{\omega}{2}} \pi} \frac{d a}{d a / d \tau} .
$$


We can find the value $d a / d \tau$ from the first of Eqs. (4.4). Taking into account that the LPT passes through zero amplitude, the expression for $\cos \delta$ can be found from energy (4.5):

$$
\cos \delta=-\frac{\sqrt{2 \omega}}{a f}\left(1-\frac{\omega}{2} a^{2}-J_{0}\left(\frac{\sqrt{2} a}{\sqrt{\omega}}\right)\right) .
$$

Finally, combining the first of Eqs. (4.4) and Eq. (4.10), and substituting the result into Eq. (4.9), we can write the period of the escape from the well as follows:

$$
T=\int_{0}^{\pi} \frac{\sqrt{2 \omega} A d A}{\sqrt{\left(-\left(\frac{\omega}{2} A\right)^{2}-J_{0}(A)+\frac{f}{2} A+1\right)\left(\left(\frac{\omega}{2} A\right)^{2}+J_{0}(A)+\frac{f}{2} A-1\right)}}
$$

(in order to write Eq. (4.11), we use the relation between the amplitude of the function $\psi$ and the amplitude of the oscillations $-a=\sqrt{\omega / 2} A)$.

The last expression can be estimated numerically at fixed values of $\omega$ and $f$. Figure 6 shows the contours of the constant escape time which have been calculated according to expression (4.11). The uncoloured region corresponds to the $(\omega-f)$ domain, where the transition to rotation of the pendulum is unreachable.

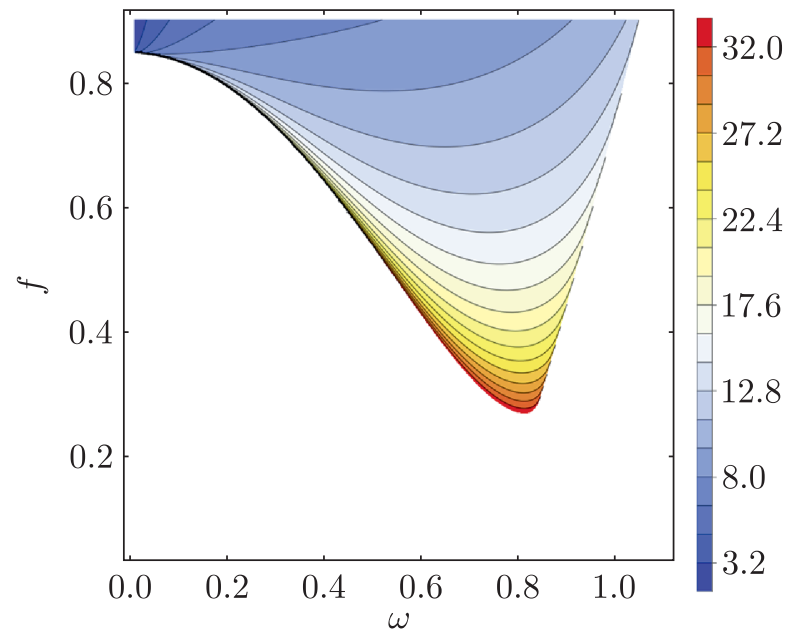

Fig. 6. The time $T$ of the escape from the potential well on the $\omega-f$ plane. The contours correspond to the constant periods of the escape from the well, which are indicated in the plot legend on the right.

\subsection{Instability of the rotation of coupled pendulums}

In this section we would like to carry out a stability analysis for the in-phase rotation of two coupled pendulums. For a more detailed description, we refer the reader to [33].

The energy of two coupled pendulums is determined as follows:

$$
H=\sum_{j=1,2}\left(\frac{1}{2}\left(\frac{d q_{j}}{d t}\right)^{2}+\sigma\left(1-\cos q_{j}\right)+\frac{\beta}{2}\left(1-\cos \left(q_{j}-q_{3-j}\right)\right)\right) .
$$

(In order to allow the mutual rotation of the pendulums, we assume a $2 \pi$-periodic interpendulum potential, which is similar to the interaction of the coaxially arranged dipoles.) 
The equations of motion have the form

$$
\frac{d^{2} q_{j}}{d t^{2}}-\beta \sin \left(q_{3-j}-q_{j}\right)+\sigma \sin q_{j}=0 ; \quad j=1,2 .
$$

Let us introduce the in-phase mode $\theta_{1}=\left(q_{1}+q_{2}\right) / 2$ and the out-of phase mode $\theta_{2}=$ $=\left(q_{1}-q_{2}\right) / 2$. The equations of motion

$$
\begin{aligned}
\frac{d^{2} \theta_{1}}{d t^{2}}+\sigma \cos \theta_{2} \sin \theta_{1} & =0, \\
\frac{d^{2} \theta_{2}}{d t^{2}}+\beta \sin 2 \theta_{2}+\sigma \cos \theta_{1} \sin \theta_{2} & =0
\end{aligned}
$$

admit the exact solutions $\left(\theta_{1}=\theta_{1}(t), \theta_{2}=0\right)$ and $\left(\theta_{1}=0, \theta_{2}=\theta_{2}(t)\right)$, respectively.

If the energy $E$ of in-phase motion exceeds the value $2 \sigma$, the pendulums undergo synchronous rotation with the period

$$
T=2 \sqrt{2} \frac{K\left(\frac{2 \sigma}{E}\right)}{\sqrt{E}},
$$

where $K$ is the complete elliptic integral of the first kind. However, the numerical simulations show that the in-phase rotation turns out to be unstable at some values of the coupling parameter $\beta$. The difference of the rotation velocities of the pendulums exhibits some periodic perturbations, an example of which is shown in Fig. 7a.

Panel (b) of Fig. 7 shows the range of the coupling parameter $\beta$ with unstable rotations of the pendulums as a function of the rotation energy. At first glance the existence of instability in the in-phase rotation contradicts the limiting cases of the extremely large and extremely small coupling. If the coupling is negligible, the pendulums are independent and no instability in the rotation occurs. On the other hand, when the coupling constant $\beta \rightarrow \infty$, two pendulums can be considered as a single pendulum with the doubled mass, and no instability occurs again.

Let us assume that the energy of the in-phase rotation is large enough $(E \gg 2 \sigma)$. In this case we can represent the obvious solution of the first of Eqs. (4.14) as follows:

$$
\theta_{1}=\omega_{r} t+\lambda \sin \omega t
$$

where $\omega_{r}=2 \pi / T$ is the rotation frequency and $\lambda=\sigma / \omega_{r}^{2} \ll 1$.

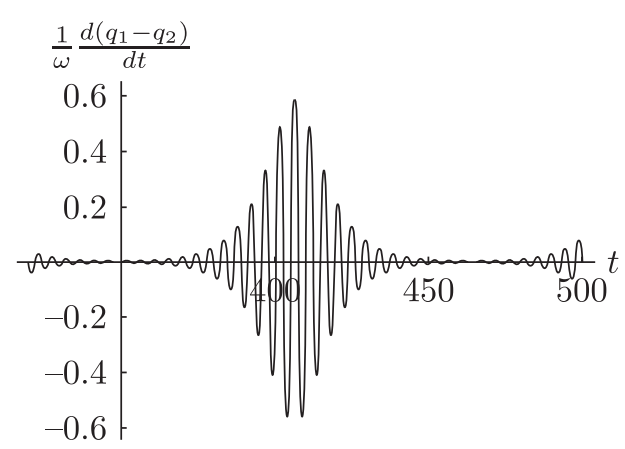

(a)

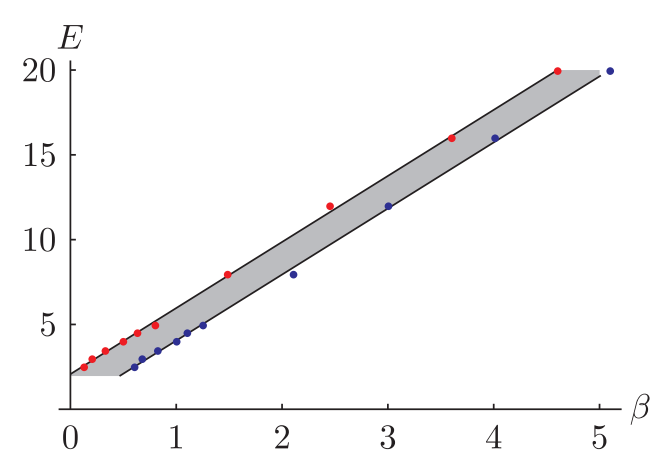

(b)

Fig. 7. (a) The data of numerical simulations of the in-phase rotation of coupled pendulums. The behavior of the difference of the pendulums' velocities in the unstable range of the in-phase rotation. (b) The range of the coupling constant $\beta(E)$, where the unstable in-phase rotation occurs, is colored gray. The blue and red points were obtained in the direct numerical simulations. The black lines show threshold values corresponding to relations (4.20) and (4.21). 
In order to analyze the stability of the in-phase rotation, we consider the out-of-phase variable as a small perturbation. Taking the solution (4.16) into account, we can write

$$
\frac{d^{2} \theta_{2}}{d t^{2}}+\left(2 \beta+\sigma \cos \omega_{r} t\right) \sin \theta_{2}=0
$$

(Taking $\theta_{2}$ as a small perturbation, we assume that $\cos \theta_{2} \approx 1$.)

Equation (4.17) is the well-known equation of the parametrically excited pendulum. It is common knowledge that the first parametric resonance occurs at a frequency that is half the eigenfrequency. Thus, we need to estimate the perturbation with frequency $\Omega=\omega_{r} / 2$.

Representing the out-of-phase perturbation in complex form according to expression (2.7), extracting the "carrier" exponential $e^{-i \Omega t}$ and discriminating the secular term, we can write stationary equations for the modulus and the phase of the perturbation:

$$
\begin{aligned}
& \frac{\sigma}{a} J_{2}\left(\sqrt{\frac{2}{\Omega}} a\right) \sin 2 \delta=0 \\
& \frac{\Omega}{2} a-\frac{1}{\sqrt{2 \Omega}} 2 \beta J_{1}\left(\sqrt{\frac{2}{\Omega}} a\right)-\frac{\sigma}{\sqrt{2 \Omega}}\left[J_{1}\left(\sqrt{\frac{2}{\Omega}} a\right)-\frac{\sqrt{2 \Omega}}{a} J_{2}\left(\sqrt{\frac{2}{\Omega}} a\right)\right] \cos 2 \delta=0,
\end{aligned}
$$

where $J_{n}$ is a Bessel function of order $n$. These equations describe the stationary out-of-phase oscillations coupled with the in-phase rotation of the pendulums. The amplitude and phase of such oscillations should be determined numerically. However, we can write the energy of such oscillations as follows:

$$
H=\frac{\Omega}{2} a^{2}-2 \beta\left(1-J_{0}\left(\sqrt{\frac{2}{\Omega}} a\right)\right)-\sigma J_{2}\left(\sqrt{\frac{2}{\Omega}} a\right) \cos 2 \delta .
$$

Taking this expression into account, we analyze the nonstationary trajectories on the phase plane for different values of the coupling parameter $\beta$.

Figure 8 shows the phase portraits of the system with rotation energy $E=5.0$ for three specific values of $\beta$. Figure 8a represents the phase plane of the system when the coupling parameter $\beta$ is smaller than the bottom threshold of the instability. One can see that there is only the stationary solution $a=0$ for any values of phase $\delta$. Any trajectories that are close to the stationary state cannot rise and the rotation is stable.

However, if the coupling constant $\beta$ exceeds some threshold, a new stationary point with $a \neq 0$ and phase $\delta=0$ appears. Simultaneously the trajectory that separates the sets of the closed and transit-time trajectories forms. It is important that this trajectory passes through the zero value of the amplitude $a$, therefore, any perturbations which start from zero amplitude evolve along this trajectory. This trajectory is the aforementioned LPT. Figure 8b shows the phase portrait after formation of the stationary solution and the LPT. It should be noted that, from the viewpoint of the rotational instability, it is not the existence of the stationary point that matters, but the appearance of the limiting phase trajectory.

In order to determine the threshold of the instability, one should note that it arises via formation of a stationary solution at the point $(a=0, \delta=0)$. This is needed in the condition $\partial^{2} H_{2} / \partial a^{2}=0$ at this point. Solving this equation with respect to the coupling parameter, we get the bottom threshold value as follows:

$$
\beta_{l}=\frac{1}{4}\left(2 \Omega^{2}-\sigma\right) .
$$




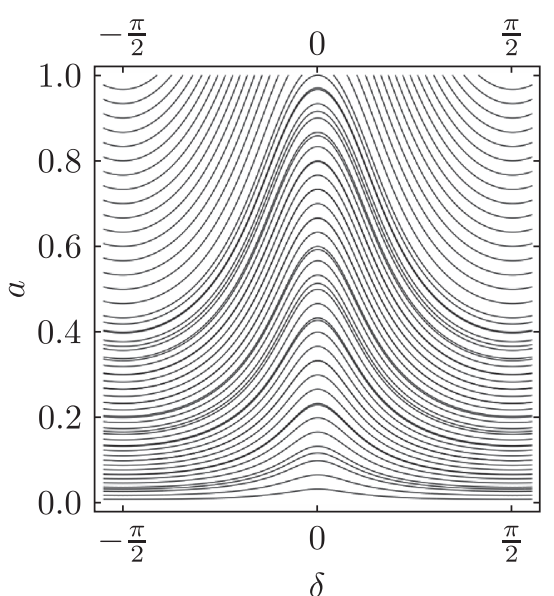

(a)

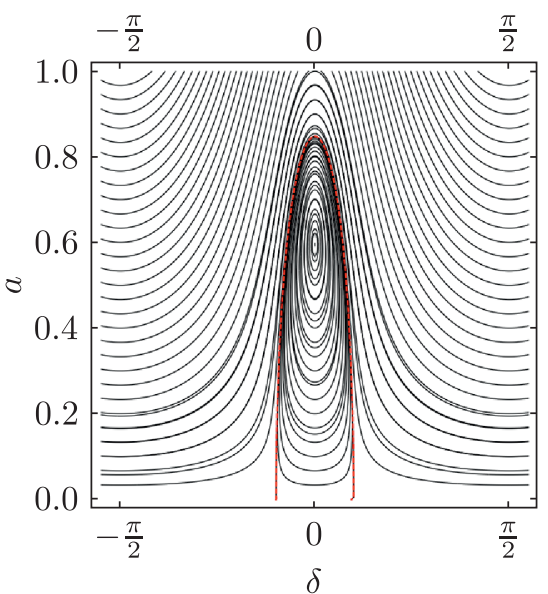

(b)

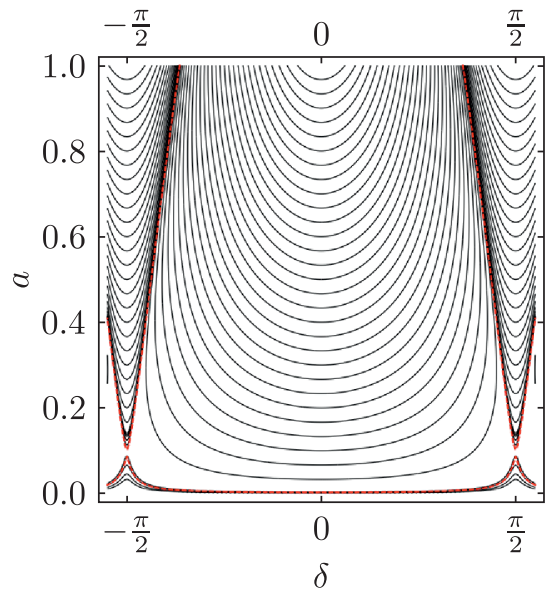

(c)

Fig. 8. (Color online) The phase plane of system (4.19) at three values of the coupling parameter $\beta$ (a) $\beta=0.7$, (b) $\beta=0.9$, (c) $\beta=1.2275$. $E=5.0, \sigma=1$.

As the coupling parameter grows, so does the amplitude of the stationary solution, as well as the LPT. However, when the LPT's low edges reach the phase value $\delta= \pm \pi / 2$, the next bifurcation occurs. Namely, the symmetric pair of the unstable stationary solutions appear at the points $(a=0, \delta= \pm \pi / 2)$. Simultaneously, the LPT transforms to the separatrix crossing these unstable states. Figure $6 \mathrm{c}$ shows the phase plane after the second bifurcation. Since the same branch of the separatrix passes through both unstable points, no small-amplitude trajectories can grow. The value of the coupling parameter corresponding to this bifurcation can be estimated from the criterion of stationary point creation with phase $\delta= \pm \pi / 2$, which leads to

$$
\beta_{t}=\frac{1}{4}\left(2 \Omega^{2}+\sigma\right) .
$$

The thresholds (4.20), (4.21) are shown in Fig. 7b by the black lines. One can notice an excellent agreement of these values with the data of numerical simulations.

The three problems discussed above exhaust the list of the examples by which we would like to illustrate the applications of the CEVA, but many other applications of the method discussed above can be found in [35]. 


\section{Conclusion}

One would like to pay attention to the two aspects of the CEVA. First of all, due to the representation of the envelopes as complex functions, which is an analogue of the procedure of secondary quantization in quantum mechanics, averaging the equations of motion leads to analytically solved relations between the amplitude and the frequency of oscillations even in the cases of essentially nonlinear systems. Therefore, we get the main-order approximation, which takes into account the nonlinear features of the system, and it turns out to be adequate in a wide range of oscillation amplitudes. Moreover, as was shown in Section 4, the main-order approximation allows one to consider the forced oscillations taking the dissipative effects into account. It is noteworthy that expression (4.3) for the stationary oscillation amplitude generalizes the result which is given in the classical book by Bogoliubov an Mitropolosky [4]. This expression shows that the resonant amplitude-frequency curve exhibits a universal profile, where the role of the eigenfrequency is played by the frequency of free nonlinear oscillations, which depends on the amplitude. We do not have to require any small parameters for the description of the main-order approximation, but in the absence of such parameters the procedure of estimating the corrections turns out to be ambiguous. The most natural assumption is that the discrepancies between exact and approximate frequencies can be corrected by allowing for higher harmonics. In this way, we should estimate the amplitudes of these harmonics in the lowest order and calculate the shift of the associated frequency. Thus, we can formulate the criterion for applicability of the single-frequency main-order solution as the relative smallness of the higher harmonics' amplitudes. However, this criterion strongly depends on the requirements of the problem to be solved. It is theoretically possible to allow not only for the first higher harmonics, but tedious calculations and complexity of numerical calculations form technical obstacles for this procedure. It should be noted that the equations that are determined by the frequency shift are nonlinear in any order on the correction amplitudes. Thus, we can say that the CEVA allows one to describe nonlinear stationary oscillations (nonlinear normal modes) even in the main order and to estimate the frequency shift which originates from the high harmonic effect.

However, the absence of a small parameter in the initial problem also makes ambiguous the development of the nonstationary dynamics of the system under consideration. Actually, due to the averaging we get the description of the system's dynamics in terms of the envelopes. According to their definition, the envelopes are functions whose time scale greatly exceeds the period of self-excited oscillations. In the absence of a small parameter it is impossible to determine what the characteristic time of the nonstationary processes is. It is clear that the scale has to be controlled by the inner features of the phenomena considered. As an example we can point to the resonant interaction between nonlinear normal modes in the onedimensional lattices, which leads to energy localization and the formation of envelope solitons (breathers) [24-26]. In such a case both the frequency gap and the oscillation amplitudes are the control parameters of the transfer of the energy along the chain. Therefore, we need an algorithm for constructing the nonstationary dynamical equations. We believe that the slow Hamiltonian (3.8), which was suggested in Section 3, makes possible an analysis of the nonstationary problem. As was defined above, the slow Hamiltonian is the difference between the averaged Hamiltonian of the original system and the energy of the single-frequency oscillations of the main-order approximation. In this case, the first variation of the slow Hamiltonian is zero for any eigen oscillations (nonlinear normal mode), but it is clear that, due to other (disturbed) oscillations, this variation will be a function of some time. The scale of this time is determined 
by the measure of deviation from the steady-state oscillations, and the slow evolution of the envelope is determined by Eq. (3.10), which can be expanded as a series in terms of small disturbances (3.12). On the other hand, we can ignore the expansion of the slow Hamiltonian if we have the possibility of solving Eq. (3.10), but in solving this equation we have to control the "slowness" of the evolution of the function $\psi$. This approach turns out to be very useful if we restrict ourselves to a qualitative analysis of the phase space which is parameterized by the frequency, as demonstrated in Section 4. In fact, the "slowness" depends greatly on the formulation and the requirements of the initial problem and, sometimes, 5 to 7 periods of the steady-state oscillations can turn out to be an adequate time for the accurate description of the slow nonstationary processes. In conclusion, we would like to remark that the CEVA admits a generalization to systems with an infinite number of degrees of freedom, as was discussed in $[26,27,35]$.

\section{A. The dispersion relation for $1 \mathrm{D}$ sine-lattice}

In the Appendix we clarify the derivation of the stationary equation in terms of complex variables. We will use the chain of interacting particles with $2 \pi$-periodic potential (the so-called sine-lattice $[26,36,37])$. The Hamiltonian function of the chain can be written as

$$
H=\sum_{j=1}^{N}\left(\frac{1}{2}\left(\frac{d \varphi_{j}}{d t}\right)^{2}+\beta\left(1-\cos \left(\varphi_{j+1}-\varphi_{j}\right)\right)+\sigma\left(1-\cos \varphi_{j}\right)\right)
$$

where $\varphi_{j}$ is the displacement of the $j$ th particle from the ground state, $N$ is the number of particles, and $\beta$ and $\sigma$ are the constants.

The corresponding equations of motion read

$$
\frac{d^{2} \varphi_{j}}{d t^{2}}-\beta\left(\sin \left(\varphi_{j+1}-\varphi_{j}\right)-\sin \left(\varphi_{j}-\varphi_{j-1}\right)\right)+\sigma \sin \varphi_{j}=0
$$

Let us expand the trigonometric functions in a Taylor series and replace the variables $\varphi_{j}$ according to expressions (2.3):

$$
\begin{gathered}
i \frac{d \Psi_{j}}{d t}-\frac{\omega}{2}\left(\Psi_{j}-\Psi_{j}^{*}\right)+\frac{1}{\sqrt{2 \omega}} \sum_{k=0}^{\infty} \frac{(-1)^{k}}{(2 k+1) !}\left(\frac{1}{\sqrt{2 \omega}}\right)^{2 k+1} \times \\
\left(\beta\left(\left(\Psi_{j+1}-\Psi_{j}+c c\right)^{2 k+1}-\left(\Psi_{j}-\Psi_{j-1}+c c\right)^{2 k+1}\right)-\sigma\left(\Psi_{j}+\Psi_{j}^{*}\right)^{2 k+1}\right)=0 .
\end{gathered}
$$

In order to find the stationary single-frequency solution, we need to represent the functions $\Psi_{j}(t)=\psi_{j} e^{-i \omega t}$ with modulus $\psi_{j}$ which does not depend on time. It is easy to show that substituting this expression into Eq. (A.3) and averaging it over period $2 \pi / \omega$ lead to the equation

$$
\begin{gathered}
\frac{\omega}{2} \psi_{j}+\frac{\beta}{\sqrt{2 \omega}}\left(J_{1}\left(\sqrt{\frac{2}{\omega}}\left|\psi_{j+1}-\psi_{j}\right|\right) \frac{\psi_{j+1}-\psi_{j}}{\left|\psi_{j+1}-\psi_{j}\right|}-\right. \\
\left.J_{1}\left(\sqrt{\frac{2}{\omega}}\left|\psi_{j}-\psi_{j-1}\right|\right) \frac{\psi_{j}-\psi_{j-1}}{\left|\psi_{j}-\psi_{j-1}\right|}\right)-\frac{\sigma}{\sqrt{2 \omega}} J_{1}\left(\sqrt{\frac{2}{\omega}}\left|\psi_{j}\right|\right) \frac{\psi_{j}}{\left|\psi_{j}\right|}=0
\end{gathered}
$$


where $J_{1}$ is a Bessel function of the first order. Assuming the periodic boundary conditions and taking relation (2.8) into account, one can see that the plane wave $\psi_{j}=\chi e^{i \kappa j}$ with $\kappa=2 \pi k / N$, $k=0,1, \ldots, N / 2$ and $\chi=\sqrt{\omega / 2} A$ is an exact solution of equation $(A .4)$ if frequency $\omega$ is determined by the expression

$$
\omega^{2}=\frac{2}{A}\left(\sigma J_{1}(A)+2 \beta J_{1}\left(2 A \sin \frac{\kappa}{2}\right) \sin \frac{\kappa}{2}\right) .
$$

\section{B. Estimation of the frequencies of the simple nonlinear oscillator}

In order to demonstrate the efficiency of the CEVA in the estimation of the amplitudefrequency relation for stationary oscillations, we performed a calculation for the simple one degree-of-freedom system:

$$
\frac{d^{2} x}{d t^{2}}+x+x^{3}+x^{5}=0 .
$$

Following the procedure of Section 2, it is easy to show that the frequency of the stationary oscillations is determined as follows:

$$
\omega_{1}=\sqrt{1+\frac{3}{4} A^{2}+\frac{5}{8} A^{4}} .
$$

We compare these results with the data which have been obtained in [39] and [40] by different methods. The final expression for the frequency in [39] was obtained by some intuitive considerations:

$$
\omega_{2}=\sqrt{1+0.83 A^{2}+0.51783 A^{4}} .
$$

In spite of that, this expression is extremely similar to Eq. (B.2), we suppose that the choice of numerical coefficients is rather intuitive [39]. The corresponding values of the oscillation frequency are shown in Table 1 as $\omega_{2}$.

The method of high-order harmonic balance was used in [40]. After some tedious calculations the values of the oscillation frequency for different amplitudes were obtained. They are represented in Table 1 as $\omega_{3}$.

The main problem of the two papers just mentioned is that even a little change in the initial equation (B.1) (for example, a variation of the numerical coefficients) can cause a hardly

Table 1. Frequencies of the stationary oscillations for system (B.1) for different amplitudes $A$. $\omega_{e}$ corresponds to the exact value, $\omega_{1}$ is determined by expression $(B .2)$, and $\omega_{2}$ and $\omega_{3}$ are reprinted from [39] and [40], respectively. Relative errors $\delta_{j}$ are calculated according to expression $(B .4)$

\begin{tabular}{cccccccc}
\hline \hline$A$ & $\omega_{e}$ & $\omega_{1}$ & $\omega_{2}$ & $\omega_{3}$ & $\delta_{1}$ & $\delta_{2}$ & $\delta_{3}$ \\
\hline 0.1 & 1.0038 & 1.0038 & 1.0042 & 1.0038 & -0.0004 & 0.0396 & 0.0003 \\
0.3 & 1.0356 & 1.0357 & 1.0387 & 1.0358 & -0.0102 & 0.3051 & 0.0226 \\
0.5 & 1.1065 & 1.1075 & 1.1135 & 1.1084 & -0.0870 & 0.6283 & 0.1635 \\
1.0 & 1.5236 & 1.5411 & 1.3718 & 1.5485 & -1.149 & 9.9639 & 1.6332 \\
3.0 & 7.2686 & 7.6404 & 7.1003 & 7.7265 & -5.114 & 2.3156 & 6.2990 \\
5.0 & 19.1815 & 20.2577 & 18.584 & 20.5097 & -5.611 & 3.1129 & 6.9244 \\
\hline \hline
\end{tabular}




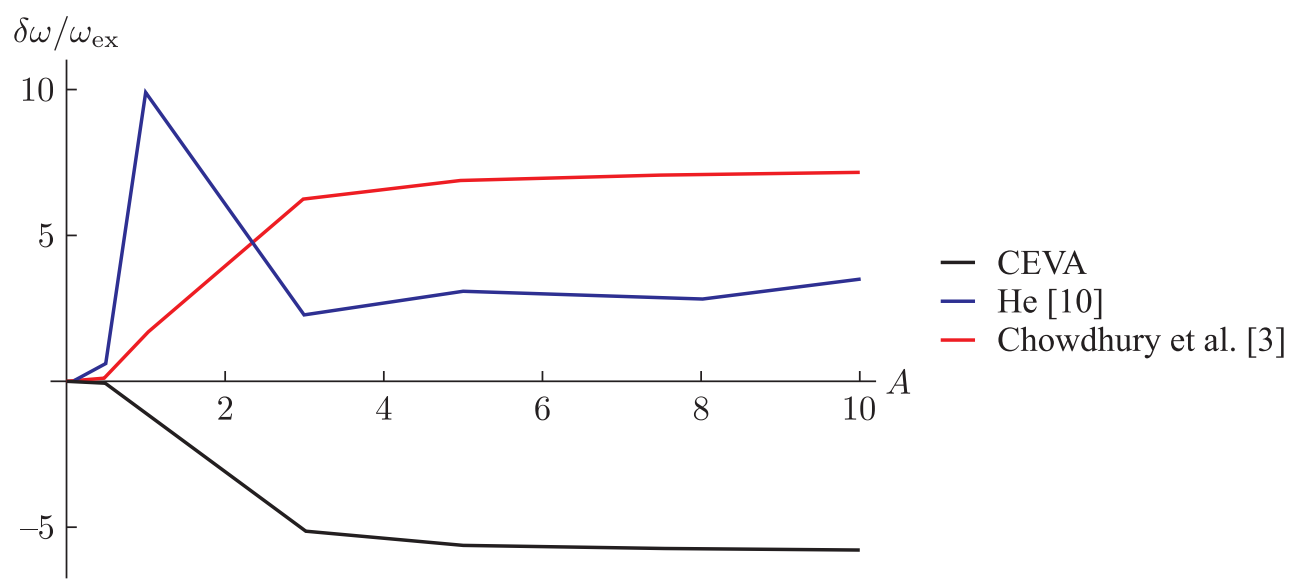

Fig. 9. Relative errors for the oscillation frequencies. The black, blue and red lines show the results of the present work and the data from [39] and [40], respectively.

predictable effect on the final result and, in order to estimate it, we need to perform a full calculation once again. Table 1 and Figure 9 present a comparative analysis of the data calculated by expressions (B.2) and reprinted from [39] and [40]. The relative errors have been calculated with respect to exact values $\left(\omega_{e}\right)$ (see Table 1$)$.

$$
\delta_{j}=\frac{\omega_{j}-\omega_{e}}{\omega_{e}} \quad(j=1,2,3) .
$$

\section{References}

[1] Nonlinear Science at the Dawn of the 21st Century, P. L. Christiansen, M. P. Sorensen, A. C. Scott (Eds.), Lecture Notes in Phys., vol. 542, Berlin: Springer, 2000.

[2] Scott, A., Nonlinear Science: Emergence and Dynamics of Coherent Structures, 2nd ed., Oxf. Texts Appl. Eng. Math., vol. 8, Oxford: Oxford Univ. Press, 2003.

[3] Sagdeev, R. Z., Usikov, D. A., and Zaslavsky, G. M., Nonlinear Physics: From the Pendulum to Turbulence and Chaos, Chur: Harwood Acad. Publ., 1990.

[4] Bogoliubov, N. N. and Mitropolsky, Yu. A., Asymptotic Methods in the Theory of Non-Linear Oscillations, New York: Gordon \& Breach, 1961.

[5] Krylov, N. M. and Bogoliubov, N. N., Introduction to Non-Linear Mechanics, Ann. of Math. Stud., vol. 11, Princeton, N.J.: Princeton Univ. Press, 1943.

[6] Nayfeh, A.H. and Mook, D. T., Nonlinear Oscillations, New York: Wiley, 1979.

[7] Sanders, J. A., Verhulst, F., and Murdock, J., Averaging Methods in Nonlinear Dynamical Systems, 2nd ed., Appl. Math. Sci., vol. 59, New York: Springer, 2007.

[8] Mickens, R. E., Truly Nonlinear Oscillators: An Introduction to Harmonic Balance, Parameter Expansion, Iteration, and Averaging Methods, Singapore: World Sci., 2010.

[9] Cveticanin, L., Strong Nonlinear Oscillators: Analytical Solutions, 2nd ed., Cham: Springer, 2018.

[10] Esmailzadeh, E., Younesian, D., and Askari, H., Analytical Methods in Nonlinear Oscillations: Approaches and Applications, Solid Mech. Appl., vol. 252, Dordrecht: Springer, 2019.

[11] He, J.-H., Some Asymptotic Methods for Strongly Nonlinear Equations, Internat. J. Modern Phys. B, 2006, vol. 20, no. 10, pp. 1141-1199.

[12] Leon, J. and Manna, M., Multiscale Analysis of Discrete Nonlinear Evolution Equations, J. Phys. A, 1999, vol. 32, no. 15, pp. 2845-2869. 
[13] Kevorkian, J. and Cole, J. D., The Method of Multiple Scales for Ordinary Differential Equations, in Multiple Scale and Singular Perturbation Methods, Appl. Math. Sci., vol.114, New York: Springer, 1996, pp. 267-409.

[14] van der Pol, B., On "Relaxation-Oscillations", The London, Edinburgh, and Dublin Philosophical Magazine and Journal of Science (7), 1926, vol. 2, no. 11, pp. 978-992.

[15] van der Pol, B. and van der Mark, J., Frequency Demultiplication, Nature, 1927, vol. 120, pp. 363-364.

[16] Manevitch, L. I., New Approach to Beating Phenomenon in Coupled Nonlinear Oscillatory Chains, Arch. Appl. Mech., 2007, vol.77, pp.301-312.

[17] Manevitch, L.I., The Description of Localized Normal Modes in a Chain of Nonlinear Coupled Oscillators Using Complex Variables, Nonlinear Dynam., 2001, vol. 25, nos. 1-3, pp. 95-109.

[18] Kosevich, A. M. and Kovalev, A.S., Introduction to Nonlinear Physical Mechanics, Kiev: Naukova Dumka, 1989 (Russian).

[19] Dirac, P. A. M., The Principles of Quantum Mechanics, 4th ed., Internat. Ser. Monogr. Phys., vol. 27, New York: Oxford Univ. Press, 1958.

[20] Manevitch, L. I. and Smirnov, V. V., Resonant Energy Exchange in Nonlinear Oscillatory Chains and Limiting Phase Trajectories: from Small to Large Systems, in Advanced Nonlinear Strategies for Vibration Mitigation and System Identification, A. F. Vakakis (Ed.), CISM International Centre for Mechanical Sciences, vol. 518, Vienna: Springer, 2010, pp. 207-258.

[21] Manevitch, L. I. and Musienko, A.I., Limiting Phase Trajectories and Energy Exchange between Anharmonic Oscillator and External Force, Nonlinear Dyn., 2009, vol. 58, pp. 633-642.

[22] Manevitch, L. I., Smirnov, V. V., and Romeo, F., Non-Stationary Resonance Dynamics of the Harmonically Forced Pendulum, Cybern. Phys., 2016, vol. 5, no. 3, pp. 91-95.

[23] Kovaleva, A. and Manevitch, L. I., Limiting Phase Trajectories and Emergence of Autoresonance in Nonlinear Oscillators, Phys. Rev. E, 2013, vol. 88, no. 2, 024901, 6 pp.

[24] Manevitch, L. I. and Smirnov, V. V., Limiting Phase Trajectories and the Origin of Energy Localization in Nonlinear Oscillatory Chains, Phys. Rev. E (3), 2010, vol.82, no. 3, 036602, 9 pp.

[25] Manevitch, L. I., Smirnov, V. V., and Romeo, F., Stationary and Non-Stationary Resonance Dynamics of the Finite Chain of Weaky Coupled Pendula, Cybern. Phys., 2016, vol. 5, no. 4, pp. 130-135.

[26] Smirnov, V. V. and Manevitch, L. I., Large-Amplitude Nonlinear Normal Modes of the Discrete Sine Lattices, Phys. Rev. E, 2017, vol. 95, no. 2, 022212, 8 pp.

[27] Smirnov, V.V., Manevitch, L.I., Strozzi, M. and Pellicano, F., Nonlinear Optical Vibrations of Single-Walled Carbon Nanotubes: 1. Energy Exchange and Localization of Low-Frequency Oscillations, Phys. D, 2016, vol. 325, pp. 113-125.

[28] Smirnov, V.V. and Manevitch, L. I., Semi-Inverse Method in Nonlinear Mechanics: Application to Couple Shell- and Beam-Type Oscillations of Single-Walled Carbon Nanotubes, Nonlinear Dyn., 2018, vol. 93, pp. 205-218.

[29] Kovaleva, M. A., Manevich, L. I., and Pilipchuk, V. N., New Type of Synchronization of Oscillators with Hard Excitation, J. Exp. Theor. Phys., 2013, vol.117, no. 2, pp. 369-377; see also: Zh. Èksper. Teoret. Fiz., 2013, vol.144, no. 2, pp. 428-437.

[30] Manevitch, L. and Kovaleva, M., Vibration Analog of a Superradiant Quantum Transition, Dokl. Phys., 2013, vol.58, no. 10, pp.428-432; see also: Dokl. Akad. Nauk, 2013, vol.452, no.5, pp. $514-517$.

[31] Bitar, D., Ture Savadkoohi, A., Lamarque, C.-H., Gourdon, E., and Collet, M., Extended Complexification Method to Study Nonlinear Passive Control, Nonlinear Dyn., 2020, vol. 99, pp. 1433-1450.

[32] Manevitch, L. I. and Vakakis, A. F., Nonlinear Oscillatory Acoustic Vacuum, SIAM J. Appl. Math., 2014, vol. 74, no. 6, pp. 1742-1762.

[33] Smirnov, V.V., Revolution of Pendula: Rotational Dynamics of the Coupled Pendula, in Problems of Nonlinear Mechanics and Physics of Materials, I. V.Andrianov, A. I. Manevich, 
Yu. V. Mikhlin, O. V. Gendelman (Eds.), Advanced Structured Materials, vol. 94, Berlin: Springer, 2019, pp. 141-156.

[34] Smirnov, L.A., Kryukov, A.K., Osipov, G. V., and Kurths, J., Bistability of Rotational Modes in a System of Coupled Pendulums, Regul. Chaotic Dyn., 2016, vol.21, nos.7-8, pp. 849-861.

[35] Manevitch, L. I., Kovaleva, A., Smirnov, V., and Starosvetsky, Yu., Nonstationary Resonant Dynamics of Oscillatory Chains and Nanostructures, Singapore: Springer, 2018.

[36] Homma, S. and Takeno, S., A Coupled Base-Rotator Model for Structure and Dynamics of DNA: Local Fluctuations in Helical Twist Angles and Topological Solitons, Prog. Theor. Phys., 1984, vol. 72, no. 4, pp. 679-693.

[37] Takeno, Sh. and Homma, Sh., A sine-Lattice (sine-Form Discrete sine-Gordon) Equation: One- and Two-Kink Solutions and Physical Models, J. Phys. Soc. Japan, 1986, vol. 55, no. 1, pp. 65-75.

[38] Gendelman, O. V. and Karmi, G., Basic Mechanisms of Escape of a Harmonically Forced Classical Particle from a Potential Well, Nonlinear Dyn., 2019, vol. 98, pp. 2775-2792.

[39] He, J. H., The Simplest Approach to Nonlinear Oscillators, Results Phys., 2019, vol. 15, 102546, 2 pp.

[40] Chowdhury, M., Hosen, M. A., Ahmad, K., Ali, M., and Ismail, A., High-Order Approximate Solutions of Strongly Nonlinear Cubic-Quintic Duffing Oscillator Based on the Harmonic Balance Method, Results Phys., 2017, vol.7, pp. 3962-3967.

[41] Gendelman, O., Targeted Energy Transfer in Systems with Non-Polynomial Nonlinearity, J. Sound Vibration, 2008, vol. 315, no. 3, pp. 732-745.

[42] Gendelman, O.V. and Manevitch, L. I., Method of Complex Amplitudes: Harmonically Excited Oscillator with Strong Cubic Nonlinearity, in ASME 2003 International Design Engineering Technical Conferences and Computers and Information in Engineering: Vol. 5: 19th Biennial Conference on Mechanical Vibration and Noise, Parts A, B, and C, Paper No: DETC2003/VIB-48586, pp. 2355-2359.

[43] Whitham, G. B., Variational Methods and Applications to Water Waves, Proc. Roy. Soc. London Ser. A, 1967, vol. 299, no. 1456, pp. 153-172.

[44] Whitham, G. B., Dispersive Waves and Variational Principles, in Nonlinear Waves, S. Leibovitch, A. R. Seebass (Eds.), Itaca/London: Cornell Univ. Press, 1974, pp. 151-180.

[45] Smirnov, V. V. and Manevitch, L. I., Dynamics of the System with Antiferromagnetic Arrangement: Classical Description, in Advanced Problems in Mechanics (St. Petersburg, 2020). 